\title{
Acoustic instabilities at the transition from the radiation-dominated to the matter-dominated universe
}

\author{
G. Siemieniec-Oziębło and A. Woszczyna
}

\author{
Astronomical Observatory, Jagellonian University, Faculty of Mathematics, Physics and Computer Science, ul. Orla 171, \\ 30-244 Kraków, Poland
}

Received 20 December 2002 / Accepted 23 February 2004

\begin{abstract}
The transition from acoustic noise in the radiation-dominated universe to the density structures in the matter dominated epoch is considered. The initial state is a stochastic field of sound waves moving in different directions. The construction of the initial state is compatible with the hyperbolic type of propagation equation for density perturbations, and parallel to the theory of stochastic background of gravitational waves. Instantaneous transition between the cosmological epochs is assumed, and Darmois-Israel joining conditions are applied to match solutions for sound waves with growing or decaying modes at the decoupling. As a result a substantial amplification of the low scale structures is obtained.
\end{abstract}

Key words. cosmology: theory - cosmology: miscellaneous - cosmology: large-scale structure of the universe

\section{Introduction}

Simple models of transitions between different cosmological epochs help us to understand amplification of scalar fields, electromagnetic and gravitational waves in the expanding universe (Frieman \& Turner 1984; Abbott \& Harari 1986; Hu 1998; Grishchuk 1996; Allen et al. 2000). Typical cosmologies of that class consist of three phases: 1) a semiclassical phase, which is commonly identified with de Sitter stage, 2) the radiation-dominated epoch described by the equation of state $p=\epsilon / 3$, and 3 ) the matter dominated era when pressure is negligible $p=0$. Transitions between them are assumed to be instantaneous. The physical meaning of these models is close to that of simple models of the particle scattering on rectangular potential barriers in quantum mechanics. Asymptotic results weakly depend on the barrier profile, so we hope, that in more realistic cosmology, the perturbation amplitude in the remote past and the far future should only marginally depend on the transition details.

For scalar or electrodynamic fields, as well as for gravitational waves the amplification can be measured by Bogolubov coefficients (Birrell \& Davies 1982). These fields are governed by hyperbolic partial differential equations in each of discussed epochs and the changes in the equation of state - the change in the background dynamics - result in a specific relations between Fourier modes in the in and out state, respectively. For some frequencies, counter-propagating waves are substantially amplified (creation of pairs of particles with opposite momentum) - the so called back scattering effect

Send offprint requests to: G. Siemieniec-Oziębło, e-mail: grazyna@oa.uj.edu.pl
(Parker 1972) comes into play ${ }^{1}$. Similar effects may occur in acoustic field (Lukash 1999). Fields enhancement on non static background is generally referred to parametric amplification (Grishchuk 1995).

Neither parametric amplification nor particle creation theories provide appropriate language to describe the growth of density perturbations in the transition to matter-dominated epoch. The scalar perturbations form waves (Sachs \& Wolfe 1967; White 1973; Field \& Shepley 1968) (in quantum phonon-approach Lukash 1980; Chibisov \& Mukhanov 1982) only in the epochs of non vanishing pressure, but they transform into non-travelling ingomogeneities (growing and decaying modes) in the matter dominated epoch $(p=0)$. The change then is two-fold: 1) the transition modifies the background dynamics; 2) the propagation equation change its differential type. Bogolubov coefficients lose their physical meaning. Yet, the general scheme of the field propagation throughout the transition epoch is the same: for the scalar or electrodynamic fields the continuity of each field and its time derivative must be satisfied at the transition, for metric perturbations (both scalar and tensor) the first and the second fundamental forms must be continuous (Darmois-Israel conditions).

Classical perturbations in the cosmological models with sharp transitions have been investigated by Kodama \& Sasaki (1984) or Hwang \& Vishniac (1991). These authors limit themselves to the standing wave solutions and to regime of low frequencies. A similar task for a universe with the radiation and dust mixture has been undertaken by Mukhanov et al. (1992)

\footnotetext{
1 For low frequency gravitational standing waves of substantial amplitude may appear (squeezed state of gravitational field, Grishchuk 1996).
} 
also in the long wave limit. In quantum theories the radiation to matter transition has been extensively discussed by Grishchuk (1994) as a part of a more complex cosmological model including an inflationary stage. Large scale perturbations where investigated there in the context CMBR temperature fluctuations. Relatively little is known about the low scale inhomogeneities.

In this paper we present exact formulae for density perturbations in the universe with sharp transition in a full range of frequencies. We start with acoustic noise in the radiation era and investigate its transition to density structures in the matter dominated epoch. We employ the autocorrelation function as a measure of structure, or equivalently its Fourier transform - the spatial spectrum of inhomogeneities. This measure agrees well with what other authors propose in cosmology. However, despite the cosmological practice we do not limit the basis of elementary solutions to "growing modes". Instead, we take into account the complete basis of Fourier modes, adequate to the hyperbolic character of the propagation equation and in full analogy to a stochastic description of gravitational waves (Allen et al. 2000) and quantum (phonon) theories (Lukash 1980; Chibisov \& Mukhanov 1982; Grishchuk 1994). Since we disregard inflation, there is no squeezing mechanism (Grishchuk 1995) for the acoustic field. We examine the spatial power spectrum without being limited to standing waves, and show that substantial amplification occurs in the high frequency regime, which has not been investigated till now.

The structure of the paper is the following: in Sect. 2 we discuss exact solutions to the perturbation equations in both radiation and matter eras employing orthogonal gauge. In the Sect. 3 we construct the 2-epoch model with the instantaneous transition in the equation of state, by use of the Darmois-Israel conditions. The Sect. 4 is devoted to the stochastic properties of the acoustic field. Finally, we investigate the time evolution of the spatial spectrum of cosmological inhomogeneities.

\section{Perturbations in the Robertson-Walker universe}

There are several independent methods to describe scalar perturbations in a gauge-invariant way (Olson 1976; Bardeen 1980; Brandenberger et al. 1983; Lyth \& Stewart 1990; Ellis \& Bruni 1989). For the universe filled with an ideal fluid ${ }^{2}$ with arbitrary non- vanishing pressure $p$ all of them lead to the same result: under appropriate choice of the perturbation variables, the propagation equations converge to the wave equation (Sachs \& Wolfe 1967; Field \& Shepley 1968; Chibisov \& Mukhanov 1982; Golda \& Woszczyna 2001). The density perturbations form travelling waves. Below we consider rotationless fluid, hence the hypersurfaces orthogonal to the fluid flow can be globally defined, and therefore, the orthogonal gauge (Lyth \& Mukherjee 1988; Lyth \& Stewart 1990; Padmanabhan 1993) is naturally applied.

The linear corrections to the energy density and the expansion rate evolve according to

$\partial_{t} \delta \epsilon(t, \boldsymbol{x})=-p_{0} \delta \vartheta(t, \boldsymbol{x})-\epsilon_{0} \delta \vartheta(t, \boldsymbol{x})-\vartheta_{0} \delta \epsilon(t, \boldsymbol{x})$

${ }^{2}$ With the diagonal energy momentum tensor $T_{\mu \nu}=(\epsilon+p) u^{\mu} u^{v}+$ $p g_{\mu v}$.

$$
\begin{aligned}
\partial_{t} \delta \vartheta(t, \boldsymbol{x})= & -4 G \pi \delta \epsilon(t, \boldsymbol{x})-\frac{\nabla^{2} \delta p(t, \boldsymbol{x})}{p_{0}+\epsilon_{0}} \\
& -\frac{2}{3} \vartheta_{0} \delta \vartheta(t, \boldsymbol{x})-\frac{\left(24 G \pi \epsilon_{0}-\vartheta_{0}^{2}\right) \delta p(t, \boldsymbol{x})}{3\left(p_{0}+\epsilon_{0}\right)}
\end{aligned}
$$

where the subscript " 0 " refers to the unperturbed, background FRW universe $\left(\epsilon_{0}=\epsilon_{0}(t)\right.$, and $p_{0}=p_{0}(t)$ are solely functions of time). The Eqs. (1) and (2) are derived from the Raychaudhuri and the continuity equations by use of the standard linearization procedure and by replacing the proper time by the orthogonal time. Below we limit ourselves to spatially flat universe $K=0$. In this case the background energy density and the expansion rate are related to each other by $8 \pi G \epsilon_{0}=\vartheta_{0}^{2} / 3$.

Let us assume now that the universe is filled by the single fluid with the equation of state $P / \epsilon=w=$ constant, where the value of $w$ determines both the evolution of the background metric (unperturbed universe) and the sound velocity for small perturbations. We express the energy density and the expansion rate as the composition of the background energy values $\epsilon_{0}, \vartheta_{0}$ and the small inhomogeneous correction $\delta \epsilon=\epsilon_{0} \delta(t, \boldsymbol{x}), \delta \vartheta=$ $\vartheta_{0} \Theta(t, \boldsymbol{x})$

$\epsilon(t, \boldsymbol{x})=\epsilon_{0}(t)(1+\delta(t, \boldsymbol{x}))$

$\vartheta(t, \boldsymbol{x})=\vartheta_{0}(t)(1+\Theta(t, \boldsymbol{x}))$

where $\delta(t, \boldsymbol{x})$ and $\Theta(t, \boldsymbol{x})$ play the role of the density and expansion contrasts, respectively. Transforming system (1), (2) to a second order propagation equation for $\delta$ we obtain a partial differential equation of the form

$$
\begin{aligned}
w^{(3)} \Delta \delta(t, \boldsymbol{x})= & \frac{\vartheta_{0}^{2}}{6}(w-1)(1+3 w) \delta(t, \boldsymbol{x}) \\
& +\vartheta_{0}\left(\frac{2}{3}-w\right) \partial_{t} \delta(t, \boldsymbol{x})+\partial_{t}^{2} \delta(t, \boldsymbol{x})
\end{aligned}
$$

with the background evolution given by $\vartheta_{0}(t)=\frac{2}{(1+w) t}$ or equivalently in the conformal time $\eta$

$$
\begin{aligned}
w^{(3)} \Delta \delta(\eta, \boldsymbol{x})= & \left(\frac{3 w-3}{3 w+1}\right) \frac{2}{\eta^{2}} \delta(\eta, \boldsymbol{x}) \\
& -\left(\frac{3 w-1}{3 w+1}\right) \frac{2}{\eta} \partial_{\eta} \delta(\eta, \boldsymbol{x})+\partial_{\eta}^{2} \delta(\eta, \boldsymbol{x}) .
\end{aligned}
$$

The conformal time $\eta$ is defined here as the integral $\int \frac{1}{a(t)} \mathrm{d} t$ of the scale factor reciprocal over the orthogonal time $t$ and ${ }^{(3)} \Delta$ stands for the Laplace operator in 3-dimensional Euclidean space. Equation (6) can be solved analytically by use of Fourier transform. Actually, we are interested in two special cases $w=1 / 3$ (radiation-filled universe) and $w=0$ (matterdomination epoch).

The propagation equations for small perturbations (5), (6) (and consequently (7) below) do not contain the gravitational constant $G$, which means that inhomogeneities do not selfgravitate unless the linear regime breaks down. They evolve as acoustic waves in the expanding gas medium (compare Sachs $\&$ Wolfe 1967; Stone 2000). All the perturbation equations obtained in different gauge invariant formalisms can be reduced to an equation of the form (5) by suitable changes of variables (and with different meaning of the variable $\delta$ and the 
time parameter) (Golda \& Woszczyna 2001). The necessary changes reads (in the notation: reference: original notation $\rightarrow$ $\delta$ ) (Sakai 1969): $K \rightarrow \delta$;

(Bardeen 1980): $\rho_{\mathrm{m}} \rightarrow \delta$;

(Kodama \& Sasaki 1984, Chap. IV): $\Delta \rightarrow \delta$;

(Lyth \& Mukherjee 1988): $\delta \rightarrow \delta$;

(Padmanabhan 1993): $\delta \rightarrow \delta$;

(Brandenberger et al. 1983): $\Phi_{\mathrm{H}} / \rho a^{2} \rightarrow \delta$;

(Ellis et al. 1990): $\mathcal{D} \rightarrow \delta$.

Transformations of these equations to conformal time (if parameterized differently) are necessary.

In the universe filled with highly relativistic matter, the scale factor $a(\eta)$ evolves as a linear function of the conformal time: $a(\eta)=\sqrt{\mathcal{M} / 3} \eta$, and preserves $\mathcal{M}=\epsilon_{0} a^{4}$ as the constant of motion. Equation (6) expressed in conformal time takes the canonical form (independent of first derivatives)

$\frac{1}{3}{ }^{(3)} \Delta \delta(\eta, \boldsymbol{x})=-\frac{2 \delta(\eta, \boldsymbol{x})}{\eta^{2}}+\partial_{\eta}^{2} \delta(\eta, \boldsymbol{x})$.

Equation (7) is essentially the same as the propagation equation for gravitational waves in the dust-filled universe (Grishchuk 1974; White 1992). It reduces to the wave equation in its normal form

$\frac{1}{3}^{(3)} \widehat{\Delta \delta}(\eta, \boldsymbol{x})=\partial_{\eta}^{2} \widehat{\delta}(\eta, \boldsymbol{x})$

for $\widehat{\delta}(\eta, \boldsymbol{x})$ defined as

$\widehat{\delta}(\eta, \boldsymbol{x})=\frac{1}{\eta} \partial_{\eta}(\eta \delta(\eta, \boldsymbol{x}))$.

The variable $\widehat{\delta}(\eta, \boldsymbol{x})$ is the orthogonal-gauge analogue to the Field-Shepley variable $H$ (Field \& Shepley 1968), or the Sachs-Wolfe variable $E$ Sachs \& Wolfe (1967). Solutions $\widehat{\delta}(\eta, \boldsymbol{x})$ and $\delta(\eta, \boldsymbol{x})$ expand into Fourier series in the way appropriate for massless scalar fields (Birrell \& Davies 1982),

$\widehat{\delta}(\eta, \boldsymbol{x})=\int\left(\mathrm{A}_{\boldsymbol{k}} \mathrm{u}_{\boldsymbol{k}(\epsilon)}(\eta, \boldsymbol{x})+\mathrm{A}_{\boldsymbol{k}}^{*} \mathrm{u}_{\boldsymbol{k}(\epsilon)}^{*}(\eta, \boldsymbol{x})\right) \mathrm{d} \boldsymbol{k}$

$\delta(\eta, \boldsymbol{x})=\int\left(\mathcal{A}_{\boldsymbol{k}} u_{\boldsymbol{k}(\epsilon)}(\eta, \boldsymbol{x})+\mathcal{A}_{\boldsymbol{k}}^{*} u_{\boldsymbol{k}(\epsilon)}^{*}(\eta, \boldsymbol{x})\right) \mathrm{d} \boldsymbol{k}$.

The modes $u_{\boldsymbol{k}(\epsilon)}(\eta, \boldsymbol{x})$ are simply $\frac{1}{\sqrt{2 \omega}} \mathrm{e}^{i(\boldsymbol{k} \boldsymbol{x}-\omega \eta)}$, while $u_{\boldsymbol{k}(\epsilon)}(\eta, \boldsymbol{x})$ can be found as

$$
\begin{aligned}
u_{\boldsymbol{k}(\epsilon)}(\eta, \boldsymbol{x}) & =\frac{1}{\eta} \int \eta \mathrm{u}_{\boldsymbol{k}(\epsilon)}(\eta, \boldsymbol{x}) \mathrm{d} \eta \\
& =\frac{1}{\sqrt{2 \omega}}\left(1+\frac{1}{i \omega \eta}\right) \mathrm{e}^{i(\boldsymbol{k x}-\omega \eta)} .
\end{aligned}
$$

The generic perturbation $\delta(\eta, \boldsymbol{x})$ is composed of travelling plane waves $u_{\boldsymbol{k}(\epsilon)}$ with decreasing amplitude. (Similar solutions are known in the theory of gravitational waves (White 1992), and scalar field Stebbins \& Veerarghavan 1993). The Fourier coefficient $A_{k}=-i \omega \mathcal{A}_{k}$ is an arbitrary complex function of the wave number $\boldsymbol{k}$, while $\delta(\eta, \boldsymbol{x})$ keeps real values. The frequency $\omega$ obeys the dispersion relation $\omega^{2}=k^{2} / 3$, hence waves of all length-scales propagate with the same phase and group velocity ${ }^{3}$ (compare Chibisov \& Mukhanov 1982;

\footnotetext{
3 This is not the case of open or closed universes, where sound is dispersed on the space curvature (Golda \& Woszczyna 2001).
}

Sachs \& Wolfe 1967; White 1973). Modes $u_{\boldsymbol{k}(\epsilon)}$, although different from the simple eikonal form, are still orthonormal in the sense of the Klein-Gordon scalar product.

In the epoch of matter dominance $m=\epsilon_{0} a^{3}$ is the constant of motion and the scale factor evolves as $a(\eta)=\frac{m \eta^{2}}{12}$. The propagation equation expressed in orthogonal gauge (in all formalism mentioned above) reads

$-\frac{6}{\eta^{2}} \delta(\eta, x)+\frac{2}{\eta} \partial_{\eta} \delta(\eta, x)+\partial_{\eta}^{2} \delta(\eta, x)=0$

Vanishing pressure implies the absence of the Laplace operator, consequently, the general solution consists of growing and decaying solutions involving two arbitrary functions of the space coordinates $f_{1}(\boldsymbol{x})$ and $f_{2}(\boldsymbol{x})$ :

$\delta(\eta, \boldsymbol{x})=f_{1}(\boldsymbol{x}) \eta^{2}+f_{2}(\boldsymbol{x}) \eta^{-3}$

This solution expands into Fourier series

$\delta(\eta, x)=\int\left(a_{k} \mathrm{e}^{i k x}+a_{k}^{*} \mathrm{e}^{-i k x}\right) \eta^{2}+\left(b_{k} \mathrm{e}^{i k x}+b_{k}^{*} \mathrm{e}^{-i k x}\right) \eta^{-3} \mathrm{~d} \boldsymbol{k}$

where the coefficients $a_{k}$ and $b_{k}$ are arbitrary complex functions of $\boldsymbol{k}$.

\section{Matching conditions in the transition epoch}

Consider now the two-epoch cosmological model composed of both, the radiation epoch (governed by the equation of state $p=$ $\epsilon / 3$ ), and the succeeding epoch of matter domination (with $p=$ $0)$. Below, the quantities related to these two epochs will appear with the indices (1) and (2), respectively. We assume that the transition between the epochs is instantaneous and occurs on the hypersurface $\Sigma$ orthogonal to the four velocity $u^{\mu}$ of the matter content.

The initial Cauchy conditions are unique and consistent on the hypersurface $\Sigma$ if the first and the second fundamental forms are equal (Darmois 1927; Hawking \& Ellis 1973)

$h_{\mu v(1)}(\Sigma)=h_{\mu v(2)}(\Sigma)$

$\chi_{\mu \nu(1)}(\Sigma)=\chi_{\mu \nu(2)}(\Sigma)$.

Subscripts (1) and (2) refer to both half-spaces divided by the surface $\Sigma$. For unperturbed background these equations imply the continuity of the scale factor $a(\eta)$ and its first derivative

$a_{(1)}(\Sigma)=a_{(2)}(\Sigma)$,

$\partial_{\eta} a_{(1)}(\Sigma)=\partial_{\eta} a_{(2)}(\Sigma)$.

In our two-epoch model the matching conditions are satisfied by

$a_{(1)}(\eta)=\sqrt{\frac{\mathcal{M}}{3}} \eta$
$a_{(2)}(\eta)=\frac{\sqrt{\mathcal{M}}\left(\eta+\eta_{\Sigma}\right)^{2}}{4 \sqrt{3} \eta_{\Sigma}}$

where $\eta_{\Sigma}$ denotes the time of the transition, and $\mathcal{M}$ is the constant of motion $\mathcal{M}=\epsilon_{0} a_{(1)}^{4}$ of the radiation filled universe. 
In the gauge-orthogonal formalism conditions ((16), (17)) can be rewritten to directly join the density and expansion perturbations in both epochs, before and after the transition. From Eqs. ((16), (17)) one can easily find that the energy density $\epsilon$ and the expansion rate $\vartheta$ are continuous on $\Sigma$. Indeed, the energy density $\epsilon$ is related to the induced curvature $R^{(3)}$ and the second fundamental form by (Hawking \& Ellis 1973)

$2 \epsilon=R^{(3)}-\left(\chi_{\mu}^{\mu}\right)^{2}-\chi_{\mu \nu} \chi^{\mu \nu}$.

The Ricci scalar $R^{(3)}$ on $\Sigma$ consists of the metric form $h_{\mu v}(\Sigma)$ and its space derivatives, so $R^{(3)}$ like $h_{\mu v}(\Sigma)$, is continuous in the transition. $\chi_{\mu \nu}$ is continuous on the strength of Eq. (17). As a consequence, Eq. (22) ensures, that $\epsilon$ is continuous. On the other hand the continuity of the expansion rate $\vartheta(t)$ comes directly from the same property of the second fundamental form $\chi_{\mu \nu}$. Eventually, after eliminating unperturbed values $\epsilon_{0}$ and $\vartheta_{0}$, the density perturbations obey

$\delta \epsilon_{(1)}(\Sigma)=\delta \epsilon_{(2)}(\Sigma)$,

$\delta \vartheta_{(1)}(\Sigma)=\delta \vartheta_{(2)}(\Sigma)$

where

$\delta \epsilon(\eta, \boldsymbol{x})=\epsilon_{0} \delta(\eta, \boldsymbol{x})$,

$\delta \vartheta(\eta, \boldsymbol{x})=-\frac{\vartheta_{0}}{P_{0}+\epsilon_{0}} \delta \epsilon(\eta, \boldsymbol{x})-\frac{1}{a\left(P_{0}+\epsilon_{0}\right)} \partial_{\eta} \delta \epsilon(\eta, \boldsymbol{x})$,

as derived from Eqs. (1) and (3). It is important to note that the time derivatives of $\epsilon$ and $\vartheta$ may jump, therefore neither $\delta \epsilon^{\prime}(\eta)$ nor $\delta^{\prime}(\eta)=(\delta \epsilon(\eta) / \epsilon)^{\prime}$ are continuous on $\Sigma$.

The starting point for the investigations of acoustic fields and the structures they produce in the two-epoch universe is the analysis of a single monochromatic wave (a single Fourier mode)

$u_{k(\epsilon, 1)}=\frac{1}{\sqrt{2 \omega}}\left(1+\frac{1}{i \omega \eta}\right) \mathrm{e}^{i(k x-\omega \eta)}$

This wave, while falling on $\Sigma$, generates on the "other side" of the transition surface a mixture of growing and decaying mode

$u_{k(\epsilon, 2)}=a_{k}\left(\frac{\eta+\eta_{\Sigma}}{\eta_{\Sigma}}\right)^{2} \mathrm{e}^{i k x}+b_{k}\left(\frac{\eta+\eta_{\Sigma}}{\eta_{\Sigma}}\right)^{-3} \mathrm{e}^{i k x}$.

The two coefficients $a_{k}$ and $b_{k}$ are uniquely determined from Eqs. (23), (24). Indeed, evaluating Eqs. (25), (26) for the perturbation ((27), (28)) and imposing the matching condition ((23), (24)) one obtains

$$
\begin{aligned}
& a_{k}=-\frac{3}{40} \frac{1}{\sqrt{2 \omega}} i \omega \eta_{\Sigma} \mathrm{e}^{-i \omega \eta_{\Sigma}} \\
& b_{k}=\frac{1}{\sqrt{2 \omega}}\left(8+\frac{8}{i \omega \eta_{\Sigma}}+\frac{12}{5} i \omega \eta_{\Sigma}\right) \mathrm{e}^{-i \omega \eta_{\Sigma}} .
\end{aligned}
$$

The frequency $\omega$ refers to the acoustic wave in the radiation epoch, and is related to the wave number $k$ by the linear dispersion relation $\omega=k / \sqrt{3}$. In the epoch of matter dominance, the perturbations lose their wave character, therefore it may be better to parameterise them by the wave number $k$, which has a well defined meaning in both epochs. Now, the modes $u_{k(\epsilon)}$ with coefficients ((29), (30)) take the form

$$
\begin{aligned}
u_{k(\epsilon, 1)}= & \frac{3^{1 / 4}}{\sqrt{2 k}}\left(1+\frac{\sqrt{3}}{i k \eta}\right) \mathrm{e}^{i k x-\frac{i k \eta}{\sqrt{3}}} \\
u_{k(\epsilon, 2)=} & \frac{3^{1 / 4}}{\sqrt{2 k}} \mathrm{e}^{i k x-\frac{i k \eta \Sigma}{\sqrt{3}}}\left(-\frac{3}{40} \frac{i k \eta_{\Sigma}}{\sqrt{3}}\left(1+\frac{\eta}{\eta_{\Sigma}}\right)^{2}\right. \\
& \left.+\left(8+\frac{8 \sqrt{3}}{i k \eta_{\Sigma}}+\frac{12}{5} \frac{i k \eta_{\Sigma}}{\sqrt{3}}\right)\left(1+\frac{\eta}{\eta_{\Sigma}}\right)^{-3}\right) .
\end{aligned}
$$

The second independent solution consists of complex conjugates of $u_{k(\epsilon, 1)}$ and $u_{k(\epsilon, 2)}$. We will include it later to restore the complete Fourier basis.

\section{Random acoustic fields}

The acoustic field in the early universe is shaped by thermodynamic or quantum phenomena acting prior to and during the radiation era. Their probabilistic nature leads to stochastic description. We restrict ourselves to stochastic processes homogeneous in the broad sense, called also weakly homogeneous processes, which keep their mean value and variance (standard deviation) invariant under translations. The twopoint autocorrelation functions for them are functions of the distance between points solely - not of these points' positions (Loeve 1963). Processes homogeneous in the broad sense have their Fourier representations (Loeve 1963; Sobczyk 1991; Yaglom 1961)

$\widehat{\delta}(\eta, \boldsymbol{x})=\int\left(\mathrm{A}_{\boldsymbol{k}} \mathrm{u}_{\boldsymbol{k}(\epsilon)}(\eta, \boldsymbol{x})+\mathrm{A}_{\boldsymbol{k}}^{*} \mathrm{u}_{\boldsymbol{k}(\epsilon)}^{*}(\eta, \boldsymbol{x})\right) \mathrm{d} \boldsymbol{k}$

where the integral is understood to be the stochastic integral, and the Fourier coefficients $A_{k}$ are random variables. Their expectation values fulfil (Sobczyk 1991; Yaglom 1961)

$E\left[\mathrm{~A}_{\boldsymbol{k}} \mathrm{A}_{k^{\prime}}^{*}\right] \sim \boldsymbol{\delta}\left(\boldsymbol{k}-\boldsymbol{k}^{\prime}\right)$

$E\left[A_{k} A_{k^{\prime}}\right]=0$

where $\boldsymbol{\delta}$ denotes Dirac's delta. Conversely, each process obeying Eqs. (34) and (35) is homogeneous in the broad sense.

The relations (34), (35) have a clear physical meaning. The first of them expresses the statistical independence of waves with different wave-vectors. The second means that phases of perturbations at any moment and any place are statistically independent. Altogether they assure statistical independence of waves moving in different directions. This stochastic process can be also expressed in terms of the $\delta(\eta, \boldsymbol{x})$ variable. With help of the random Fourier coefficients $\mathcal{A}_{k}$ satisfying

$E\left[\mathcal{A}_{k} \mathcal{A}_{\boldsymbol{k}^{\prime}}^{*}\right]=P_{k} \boldsymbol{\delta}\left(\boldsymbol{k}-\boldsymbol{k}^{\prime}\right)$

$E\left[\mathcal{A}_{k} \mathcal{A}_{k^{\prime}}\right]=0$

we write

$\delta[\eta, \boldsymbol{x}]=\int\left(\mathcal{A}_{\boldsymbol{k}} u_{\boldsymbol{k}(\epsilon)}(\eta, \boldsymbol{x})+\mathcal{A}_{\boldsymbol{k}}^{*} u_{\boldsymbol{k}(\epsilon)}^{*}(\eta, \boldsymbol{x})\right) \mathrm{d} \boldsymbol{k}$.

We assume that the power spectrum of the acoustic noise $P_{k}$ depends solely on the magnitude of the wave vector and 
not on its direction. The perturbation is generic - no additional constraints, nor any ordering or squeezing mechanisms have been introduced. The construction of a random acoustic field is identical with the construction of random field of gravitational waves in the matter dominated universe (Abbott \& Harari 1986; Allen 1996; Allen \& Romano 1999; Maggiore 2000). It reflects the equivalence of the propagation equations for both these classes of perturbation. Stochastic acoustic field fulfilling conditions (36), (37) can be understood as a classical limit of the quantum phonon approach (Lukash 1980; Chibisov \& Mukhanov 1982; Grishchuk 1994).

Modes $u_{k(\epsilon)}$ while regularly extended across the transition hypersurface $\Sigma$ define a stochastic structure (by means of integral (38)) in the matter dominated universe. The commonly used measure of this structure is the two-point autocorrelation function

$R(\eta, h)=\frac{1}{4 \pi} \int E[\delta(\eta, \boldsymbol{x}) \delta(\eta, \boldsymbol{x}+\boldsymbol{h})] \delta(\boldsymbol{h} \cdot \boldsymbol{h}-1) \mathrm{d} \boldsymbol{h}$

defined on the constant time hypersurfaces. Under conditions (34)-(37) this function reads

$$
\begin{aligned}
R(\eta, h) & =\frac{1}{4 \pi} \int 2 u_{\boldsymbol{k}} u_{\boldsymbol{k}}^{*} \mathcal{P}_{k} \exp (i \boldsymbol{k} \cdot \boldsymbol{h}) \boldsymbol{\delta}(\boldsymbol{h} \cdot \boldsymbol{h}-1) \mathrm{d} \boldsymbol{k} \mathrm{d} \boldsymbol{h} \\
& =\int_{0}^{\infty} 4 \pi k^{2} \frac{\sin (h k)}{h k} p_{k}(\eta) \mathrm{d} k
\end{aligned}
$$

where

$$
p_{k}(\eta)=2 u_{k(\epsilon)} u_{k(\epsilon)}^{*} P_{k}=\tau_{(\epsilon)} P_{k}
$$

plays the role of the structure spatial spectrum and $u_{k(\epsilon)}(\eta, x)$ are modes defined by Eqs. (31) and (32) extended to both epochs before and after the transition. In this way acoustic noise in the radiation era with a given spectrum $P_{k}$ determines uniquely (via Darmois-Israel conditions) the spatial spectrum of inhomogeneities $p_{k}(\eta)$ at any stage of the structure formation process.

It is important to clearly distinguish between $p_{k}$ and $P_{k}$, and understand their roles in the cosmological context. As defined above, $p_{k}$ is the Fourier transform of the two-point autocorrelation function. In the cosmological literature it is called the power spectrum ${ }^{4}$ by analogy to similar concept known in the analysis of time series (Anderson 1971), but its physical dimension is different. On the other hand, $P_{k}$ defined by Eq. (36) is the genuine power spectrum of the acoustic field (with the same physical sense as the Planck power spectrum of the electromagnetic radiation), and can be obtained from Hamiltonian description (Lukash 1980). Although in cosmology one cannot directly observe $P_{k}$, this quantity defines the physical state of acoustic field. This is $P_{k}$ not $p_{k}$, which should be either guessed, or inferred from fundamental laws of physics (Chibisov \& Mukhanov 1982). The shape of $P_{k}$ is not precised in this paper.

The time factor $\tau_{(\epsilon)}=2 u_{k(\epsilon)} u_{k(\epsilon)}^{*}$ converts the acoustic spectrum $P_{k}$ into the spatial-spectrum $p_{k}(\eta)$. (It is numerically equal to $p_{k}(\eta)$ for the white noise acoustic field: $P_{k}=$ const.).

\footnotetext{
${ }^{4}$ In this context we consequently use the name spatial power spectrum.
}

Factor $\tau_{(\epsilon)}$ contains the entire time dependence of cosmological inhomogeneities. Employing extended modes (31), (32), we find

$$
\begin{aligned}
\tau_{(\epsilon, 1)}= & \frac{\sqrt{3}}{k}\left(1+\frac{1}{\tilde{k}^{2} \tilde{\eta}^{2}}\right) \\
\tau_{(\epsilon, 2)}= & \frac{\sqrt{3}}{k}\left(\frac{128}{5}\left(1+\frac{5}{2 \tilde{k}^{2}}+\frac{9 \tilde{k}^{2}}{40}\right)(1+\tilde{\eta})^{-6}\right. \\
& \left.+\frac{3\left(10-3 \tilde{k}^{2}\right)}{25}(1+\tilde{\eta})^{-1}+\left(\frac{3 \tilde{k}}{40}\right)^{2}(1+\tilde{\eta})^{4}\right)
\end{aligned}
$$

where $\tilde{\eta}$ is the normalized time parameter $\tilde{\eta}=\eta / \eta_{\Sigma}$ and the $\tilde{k}$ is the modified wave number $\tilde{k}=k \eta_{\Sigma} / \sqrt{3}$ which measures the number of oscillations within the sound horizon on $\Sigma$. We hold the factor $\sqrt{3} / k$ in both $\tau_{(\epsilon, 1)}$ and $\tau_{(\epsilon, 2)}$ to keep modes $u_{\boldsymbol{k}(\epsilon, 1)}$ orthonormal in the sense of the Klein-Gordon scalar product in the radiation era. It is easy to check that the time factor $\tau$ is continuous on $\Sigma$.

In the radiation era, perturbations greater than the sound horizon decrease, while the low scale ones maintain constant amplitude. This can be seen directly from Eq. (42). The result qualitatively agrees with quantum theories (see Mukhanov et al. 1992 Part III, formula 20.6). Quantitative difference comes from the different gauge choice ${ }^{5}$. It should be emphasized that the spectrum $P_{k}$, the spatial spectrum $p_{k}$ and the parameter $\tau$ are invariant under unitary transformations. Therefore, the result is physically well defined - it is not the effect of any particular choice of the Fourier basis.

In the matter era the perturbations (32) are a specific mixture of growing and decaying solution (compare Ellis et al. 1990; Liang 1977). Therefore, despite vanishing pressure, their evolution depend on their length-scales. The dominant growing term $(1+\tilde{\eta})^{4}$ in Eq. (43) is multiplied by $\tilde{k}^{2}$, which means strong amplification of short waves.

A peculiar velocity field can be measured by the deviation $\delta \vartheta$ from the homogeneous Hubble flow $\vartheta_{0}=3 H$. Modes of the fluid compression ${ }^{6}$ are associated to the density modes (12), (27). On the strength of Eq. (26) we obtain

$\delta \vartheta_{(1)}=\frac{1}{\sqrt{2 \omega}} \frac{3 \sqrt{3}}{2 \sqrt{\mathcal{M}} \eta^{2}}\left(1+\frac{1}{i \eta \omega}+\frac{i \eta \omega}{2}\right) \mathrm{e}^{i(\boldsymbol{k} x-\eta \omega)}$.

$\delta \vartheta$ play a role in the matching conditions, as the Darmois-Israel conditions require that both the density and expansion fields be continuous at the transition. (Their time derivatives my undergo discontinuity). The expansion contrast $\Theta=\delta \vartheta / \vartheta_{0}$ can be expressed as a stochastic integral

$\Theta(\eta, \boldsymbol{x})=\int\left(\mathcal{A}_{\boldsymbol{k}} u_{\boldsymbol{k}(\vartheta)}(\eta, \boldsymbol{x})+\mathcal{A}_{\boldsymbol{k}}^{*} u_{\boldsymbol{k}(\vartheta)}^{*}(\eta, \boldsymbol{x})\right) \mathrm{d} \boldsymbol{k}$

5 The quadratic behaviour of the decreasing term is characteristic for the orthogonal gauge. In the synchronous system of reference this term decays as $\frac{1}{\tilde{k}^{4} \tilde{\eta}^{4}}$. It can be easily derived in the Field-Shepley formalism, see formula (5.3) of (Chibisov \& Mukhanov 1982) after substituting solutions of Eq. (4.7) and evaluating the integral over $\eta$. Equivalently, it can be proved directly in the original Lifshitz formalism (Golda \& Woszczyna 2001).

${ }^{6}$ The orthogonal gauge realizes the comoving system of reference, where the four velocity is globally chosen as $u=(1,0,0,0)$, therefore one cannot describe the peculiar velocity field directly by $\delta u$. 
with the same coefficients $\mathcal{A}_{k}$ as in Eq. (11), but with a different modes $u_{k(\vartheta)}$, given by

$$
\begin{aligned}
u_{\boldsymbol{k}(\vartheta, 1)}= & \frac{3^{1 / 4}}{2 \sqrt{2 k}}\left(1+\frac{\sqrt{3}}{i k \eta}+\frac{1}{2} \frac{i k \eta}{\sqrt{3}}\right) \mathrm{e}^{i k x-i k \frac{\eta}{\sqrt{3}}}, \\
u_{\boldsymbol{k}(\vartheta, 2)}= & \frac{3^{1 / 4}}{\sqrt{2 k}} \mathrm{e}^{i k x-\frac{i k \eta_{\Sigma}}{\sqrt{3}}}\left(\frac{i k \eta_{\Sigma}}{40 \sqrt{3}}\left(1+\frac{\eta}{\eta_{\Sigma}}\right)^{2}\right. \\
& \left.+\left(4+\frac{4 \sqrt{3}}{i k \eta_{\Sigma}}+\frac{6 i k \eta_{\Sigma}}{5 \sqrt{3}}\right)\left(1+\frac{\eta}{\eta_{\Sigma}}\right)^{-3}\right) .
\end{aligned}
$$

In close analogy with the density perturbations, one can express the peculiar velocities (perturbation in the expansion rate) in terms of the spatial autocorrelation or spectrum. This time

$$
R(\eta, h)=\frac{1}{4 \pi} \int E[\Theta(\eta, \boldsymbol{x}) \Theta[\boldsymbol{x}+\boldsymbol{h}, \eta)] \boldsymbol{\delta}(\boldsymbol{h} \cdot \boldsymbol{h}-1) \mathrm{d} \boldsymbol{h}
$$

and the time factor $\tau$ is given by formula (41) after replacing $u_{k(\epsilon)}$ by $u_{k(\vartheta)}$

$$
\begin{aligned}
\tau_{(\vartheta, 1)}= & \frac{\sqrt{3}}{4 k}\left(\frac{\tilde{\eta}^{2} \tilde{k}^{2}}{4}+\frac{1}{\tilde{\eta}^{2} \tilde{k}^{2}}\right) \\
\tau_{(\vartheta, 2)}= & \frac{\sqrt{3}}{k}\left(\frac{32}{5}\left(1+\frac{5}{2 \tilde{k}^{2}}+\frac{9 \tilde{k}^{2}}{40}\right)(1+\tilde{\eta})^{-6}\right. \\
& \left.-\frac{\left(10-3 \tilde{k}^{2}\right)}{50}(1+\tilde{\eta})^{-1}+\left(\frac{\tilde{k}}{40}\right)^{2}(1+\tilde{\eta})^{4}\right) .
\end{aligned}
$$

Unlike the density spectrum, the spectrum of the expansion rate monotonically increases in the radiation dominated universe $\left(49\right.$, see also the behaviour of modes $\left.u_{\boldsymbol{k}(\vartheta, 1)}-46\right)$. This is so, because the decrease in peculiar velocities is less than the decrease in homogeneous Hubble flow. Increase of the expansion contrast $\Theta$ with time does not invalidate the acoustic approximation, as long as the gas velocities are small when compare with the sound velocity (Whitham 1974). In our case the acoustic approximation is valid when the contribution of terms quadratic in $\delta \vartheta(\eta)$ to $\delta \vartheta^{\prime}(t)$ Eq. (2) is negligible. For large values of time this is assured by Eq. (44). Therefore, a perturbation initiated as an acoustic perturbation remains acoustic during the entire radiation era, even though $\delta \vartheta / \vartheta$ increases.

To express the perturbation enhancement relative to its initial amplitude we introduce the ratio

$T_{\epsilon}\left(\tilde{\eta}, \tilde{\eta}_{i}, \tilde{k}\right)=\frac{p_{k}(\tilde{\eta})}{p_{k}\left(\tilde{\eta}_{i}\right)}=\frac{\tau_{\epsilon}(\tilde{\eta}, \tilde{k})}{\tau_{\epsilon}\left(\tilde{\eta}_{i}, \tilde{k}\right)}$.

The so called transfer function $\delta / \delta_{i}$ is often used in a similar context. Fitting to this traditional name we will call $T(\eta, k)$ the spectrum transfer function. Contrary to $\delta / \delta_{i}$ the spectrum transfer function $T$ is real and invariant under unitary transformations of Fourier bases. Plotted on a logarithmic scale (Figs. 1 and 2) it shows that the perturbations evolution is highly sensitive to $\tilde{k}$. In both Figs. 1 and 2 we start from $\eta_{i}=0.1 \eta_{\Sigma}$ ( $\left.\tilde{\eta}_{i}=0.1\right)$ to evaluate perturbation up to $\eta=3 \eta_{\Sigma}(\tilde{\eta}=3)$.

As already mentioned, the large scale inhomogeneities (those, which are larger than the local sound horizon $\tilde{k} \ll 1$ ) decay in the radiation era $(\tilde{\eta}<1)$. In this case, the term $\frac{1}{\tilde{k}^{2} \tilde{\eta}^{2}}$ is dominant in the time factor $\tau_{(\epsilon, 1)}(\tilde{\eta}, \tilde{k})$ and strongly decreases $\log \left(T_{\varepsilon}\right)$

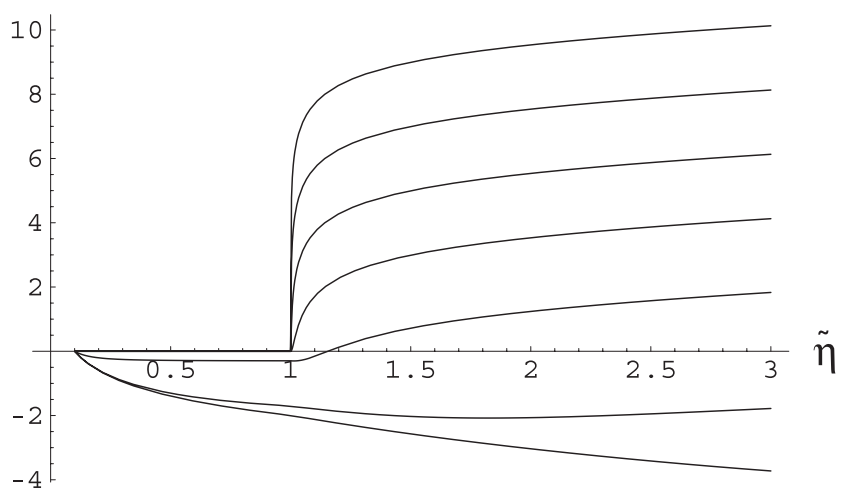

Fig. 1. The spectrum transfer function $T_{\epsilon}$ for the density perturbations (log scale) as a function of conformal time $\tilde{\eta}$. The family of solutions covers the wave numbers range $\log (\tilde{k}) \in[-1,5]$ i.e. the lowest curve on the diagram refers to the perturbation scale ten times larger than the sound horizon distance, while the top one to a perturbation scale $10^{-5}$ times smaller. Point $\tilde{\eta}=1$ represents the transition from the radiation to the matter dominated universe.

$\log \left(T_{\varepsilon}\right)$

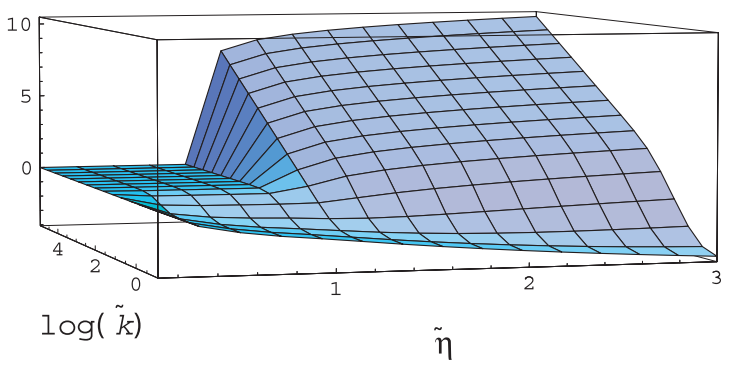

Fig. 2. The density spectrum transfer function $T_{\epsilon}$ as a function of both the conformal time $\tilde{\eta}$ and the wave number $\tilde{k}(\tilde{\eta}=1-$ the transition from the radiation to the matter dominated universe).

with time. Decay of the large scale component is a generic feature of acoustic noise on the expanding radiation dominated homogeneous background. In other words, the homogeneity of the radiation filled universe is a stable property at least as long as the generic perturbations are taken into account. Similar phenomenon of large-scale wave extinction is observed in scalar field theory (Stebbins \& Veerarghavan 1993). Although the large-scale inhomogeneities change substantially during the radiation era, their response to the change in the equation of state is very weak. Their wave character vanishes at $\eta_{\Sigma}$, but the spatial spectrum itself is insensitive to the transition (see Fig. 2).

The low scale perturbations produce a different scenario. Their amplitudes kept constant during the radiation dominatedepoch to increase by several orders of magnitude at the transition. For inhomogeneities of galactic scale $\left(M=10^{11} M_{\mathrm{s}}\right)$ the time factor $\tau$ is of $10^{8}$, which means a $10^{4}$ times amplitude enhancement. The pressure discontinuity excites low-scale perturbations and their contribution to the spatial-spectrum becomes dominant shortly after the transition.

As opposed to the density perturbations, the velocity magnitude does not change significantly at the transition. An analogue of the transfer function $T_{\vartheta}(\tilde{\eta}, \tilde{k})=\tau_{\vartheta}(\tilde{\eta}, \tilde{k}) / \tau_{\vartheta}\left(\tilde{\eta}_{i}, \tilde{k}\right)$ constructed for the expansion spectrum is shown in Fig. 3. 


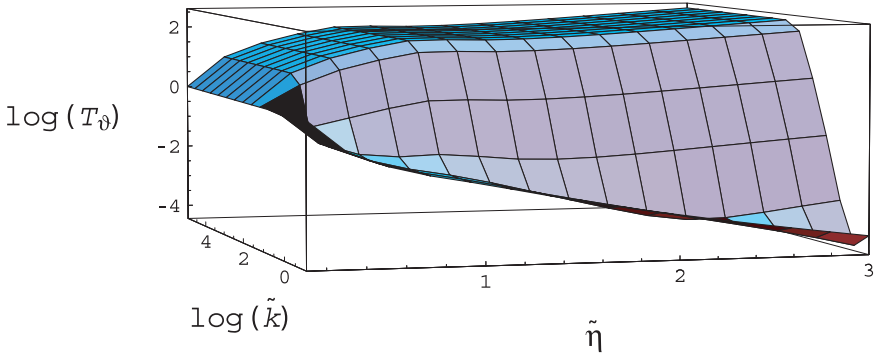

Fig. 3. The expansion spectrum transfer $T_{\vartheta}$ as a function of the conformal time $\tilde{\eta}$ and the wave number $\tilde{k}$.

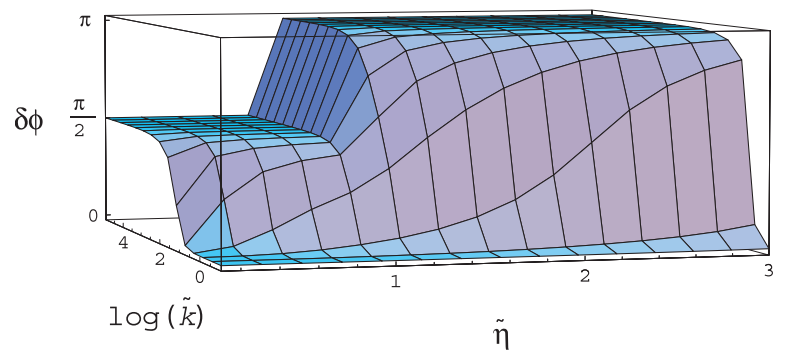

Fig. 4. Phases of perturbations.

The expansion contrast increases systematically during the radiation era and smoothly enters the matter era, keeping nearly the same growth rate before and after the transition. A substantial change in the velocity field relates to its phase. Figure 4 shows the difference in arguments of the density and expansion modes $\delta \phi=\arg \left(u_{k(\epsilon)}\right)-\arg \left(u_{k(\vartheta)}\right)$. In the radiation era large frequency modes, (both $u_{k(\epsilon)}$ and $u_{k(\vartheta)}$ ) are shifted to each other by $\pi / 2$ (compare also Eqs. (46) and (27)). This effect is the generic perturbation property in the radiation dominated fluid (Ellis et al. 1990), and is an artefact of their acoustic character. Similar relation of phases can be also obtained on the ground of binding energy analysis (Liang 1977). After the transition the arguments of the density and velocity modes differ by $\pi$ so their maxima are anti-correlated. Smallest expansion occurs in the regions of highest density. Decreasing modes have decayed and fluid flow becomes potential (Peebles 1980). (Slightly more complex is the behaviour of the low frequency modes. Solutions like that hardly achieve the phase of potential flow (the argument shift is different from $\pi$ ).)

From the hydrodynamic point of view, the transition from the radiation to the matter-dominated epoch breaks down the acoustic approximation. The sound velocity instantly falls from $v=1 / \sqrt{3}$ to zero, and in consequence, the fluid velocity formally becomes greater than the sound velocity at each point in space. (In this way the structure formation has some aspects characteristic for formation of acoustic shocks - compare Montenegro et al. 1999; Khoperskov \& Khrapov 1999). The growth of inhomogeneities is based on acoustic instability - the self-gravitation processes do not switch on until the linear regime fails. In both cases - the density and expansion fields - the spectrum transfer function monotonically increases with the wave number $k$. In particular the phenomenon of acoustic peaks is absent ${ }^{7}$. The absence of peaks is characteristic feature of the random field composed of the statistically independent moving waves (Grishchuk 1995). The spectrum transfer function may substantially change in the small $k$-regime when the universes undergoes more than one phase transition (some classical ones, like the change in the sound velocity, or semiclassical like the transition from inflationary to radiation dominated universe). In these cases the squeezed states in the acoustic field appear on large scales, which are an alternative explanation of the CMBR temperature spectrum (Bose \& Grishchuk 2002).

\section{Growing modes versus complete solutions}

When considering instantaneous transition to the matter dominated universe authors limit themselves to the large scale perturbation regime. In this regime the growing mode amplitude after the phase transition is entirely dominated by that before the phase transition and there is no chance of generating a growing mode out of a decaying mode (Kodama \& Sasaki 1984). Although the short perturbations do not produce the same scenario ${ }^{8}$ the decaying modes of any length-scale are commonly neglected at the beginning of the matter dominated era.

By abandoning all the decaying terms in the formula (43) one obtains $\tau_{\epsilon} \propto(1+\tilde{\eta})^{4}$, and consequently, the spatial spectrum of the density contrast

$p_{k}(\tilde{\eta})=T_{\epsilon}\left(\tilde{\eta}, \tilde{\eta}_{i}\right) p_{k}\left(\tilde{\eta}_{i}\right)$

divides into time dependent

$T_{\epsilon}\left(\tilde{\eta}, \tilde{\eta}_{i}\right)=\left(\frac{1+\tilde{\eta}}{1+\tilde{\eta}_{i}}\right)^{4}$

and the scale dependent $p_{k}\left(\tilde{\eta}_{i}\right)$ (given at some $\tilde{\eta}_{i}>1$ ). The spectrum transfer function $T_{\epsilon}$ drawn in the logarithmic scale is presented in Fig. 5. $T_{\epsilon}$ does not depend on $k$, therefore the perturbation is amplified in the scale-independent way. This scaleindependence is commonly attributed to the dust-filled universe $(p=0)$, but actually it requires more restrictive conditions: the perturbation must be solely composed of growing modes.

While the pure growing modes increase ten times in the interval $\tilde{\eta} \in(1,3)$, the mixture of growing and decaying low scale modes is enhanced by several orders of magnitude (compare Figs. 1 and 5). The enhancement of this mixture depends on the wave number $k$. Substantial magnification of the low scale modes (Fig. 1) means that the low-scale structures may enter the nonlinear regime first.

\footnotetext{
7 The same effect appears in more sophisticated transition models investigated numerically (Press \& Vishniac 1980), if they include the complete basis of solutions. On the other hand, as shown in (Voglis 1986), peaks may appear in a pure radiation-filled universe model without evoking complicated recombination processes, if one limits to growing modes (standing waves) with specific phase correlation. These phenomena have also been discussed in (Fang \& Wu 1996; Riazuelo \& Deruelle 2000).

8 (...) the general relation among the amplitudes (...) would be too complicated to extract any physical information out of it (Kodama \& Sasaki 1984).
} 


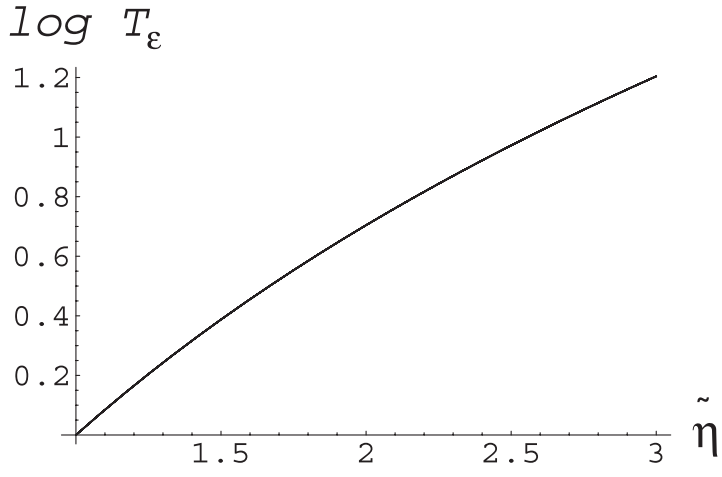

Fig. 5. The density spectrum transfer function for perturbations composed of pure growing modes.

When the decaying modes are taken into account the scaleindependence breaks down. Decaying modes "remember" perturbations' past. In our case the admixture of decaying modes defined by the joining conditions (23), (24) is an imprint of the acoustic (travelling wave) character of the density perturbations in the radiational era. Consequently, the radiational era affects the structure formation processes occurring latter on, when matter dominates. Instability of perturbations is the genuine feature of the entire cosmological evolution rather than the property of separate cosmological epochs.

\section{Observables and observational constraints}

Although our primary goal is to discuss the role of decaying solutions in the formation of the small-scale structures, it is worth checking the models consistence with data presently available for larger scales.

Data coming from the large galaxy surveys (SDSS, 2dFGRS and others) probe the large-scale mass distribution at late epochs, while the WMAP experiment reports the density fluctuations at the last scattering surface. These two categories of data potentially allow reconstruction of the spectrum transfer function i.e. the scale of inhomogeneity enhancement after decoupling.

To examine the spectrum transfer function (51) we focus on the LSS spectral estimations, with the scale range of some $100 \mathrm{Mpc}$, that at the same time overlaps the right end of the range $\left(k \sim\right.$ few $\left.10^{-1} \mathrm{Mpc}^{-1}\right)$ probed by WMAP experiment. As an example we take two close length-scales, $k \sim 0.1 \mathrm{Mpc}^{-1}$ and $k \sim 0.05 \mathrm{Mpc}^{-1}$, for which the values of $\delta$ contrast (Fig. 3 in Wu et al. 1999) are respectively, $\sim 10^{-2}$ and $\sim 10^{-3}$. These scales correspond to $l \sim 1800,700$ in the CMB spectrum and may be considered as the intermediate length-scales. The spectrum transfer function $T_{\epsilon}$ in this scales is given in Fig. 6 . The curves are drawn for the constant $\eta$-surfaces between $\eta \sim 2$ (the lowest) and $\eta-31$ - corresponding roughly to redshift $\sim 0$. The increase of the model spectrum transfer function (51) are roughly of the order of $10^{3}$ and $10^{2}$ at the scales under discussion, and reproduce the current density contrast, from the initial density contrast of the order $\sim 10^{-5}$. In spite of the high idealization, we obtain rough agreement with observations in the $k \leq 1 \mathrm{Mpc}^{-1}$ regime.

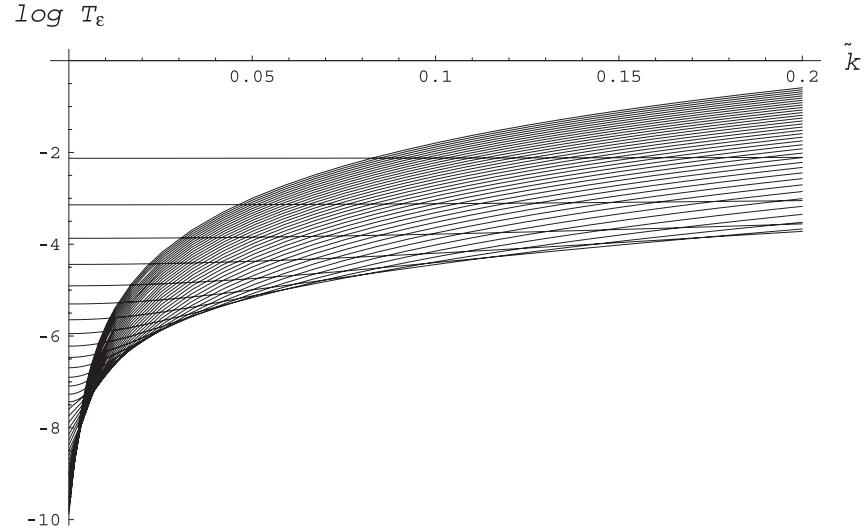

Fig. 6. The spectrum transfer function $T_{\epsilon}$ as a function of $\tilde{k}$ for diffrent $\eta \in(2,31)$

Spectrum transfer function is substantially more difficult view in the context of extremely low, or extremely large scales. In the large scale limit the spectrum transfer function (51) becomes insensitive to changes in the equation of state. This confirms that the large scale perturbations are "fossils" of the past, which is interesting in the context of their quantum or semiclassical origin. The recently reported (e.g. Oliveira-Costa et al. 2003; Hannestad 2003) discrepancy between the WMAP observational spectrum and that coming from the inflationary paradigm for scales $k \leq 0.001 \mathrm{Mpc}^{-1}$ challenges some revisions of the initial perturbation spectrum theory. On the other hand the interpretation of measurements also deserves careful examination. The perturbations and the resulting temperature fluctuations are commonly related to each other by means of the Sachs-Wolfe formula - an integral over gravitational potential performed along the photon path (Sachs \& Wolfe 1967; Peebles 1980). The formula is true in the absence or neglect of the decaying modes at the last scattering surface (Sachs \& Wolfe 1967). For the density perturbation matched to the acoustic field the decaying modes cannot be neglected, particularly the low multipoles, where discrepancies become substantial ${ }^{9}$. To understand properly the measured $C_{1}$ coefficients at low-1 limit we need the exact formula for the temperature fluctuation. (In the discussed model the exact formula can be found, but is beyond the scope of this paper.)

Violent formation of fine scale structures in the transient epoch, the characteristic feature of the model, enables inhomogeneities to enter the nonlinear regime soon after decoupling. The model qualitatively supports observations of highly developed structures at high redshifts $z$. However, the WMAP angular resolution limit rules out any quantitative estimations for the structures less than $10^{4} M_{\mathrm{gal}}$. Expecting more relevant data from the forthcoming Planck mission we anticipate, on the strength of the spectrum transfer function (51), that the CMBR temperature fluctuations in the fine-scale regime may have relatively low amplitude.

\footnotetext{
9 To neglect decaying modes one needs $\left(b_{2}\right)^{2} \ll\left(a_{2}\right)^{2}$. After employing (30) this condition is $1+40 /\left(3 k^{2} \eta_{\Sigma}^{2}\right)+100 /\left(k^{4} \eta_{\Sigma}{ }^{4}\right) \ll 1$, and is false for any $k$. Particularly, in the $k \rightarrow 0$ the error becomes arbitrarily large.
} 


\section{Summary}

Generic density perturbations in the radiation-dominated universe propagate in the same way as sounds propagate in air or electromagnetic waves in vacuum. As shown by Sachs \& Wolfe (1967) and White (1973) perturbations form waves travelling with the same speed $v=\frac{1}{\sqrt{3}}$ independently of their scales or profiles, hence gravitationally bound structures cannot form. Perturbations do not self-gravitate in the linear regime, so gravity may affect their evolution merely by affecting the dynamics of the homogeneous background. The wave character of the density perturbations (independent of their scales) is confirmed in the Hamiltonian formalism (Lukash 1980; Chibisov \& Mukhanov 1982).

The hyperbolic type of propagation equation requires appropriate perturbation statistics, where acoustic waves travelling in different directions are statistically independent. This kind of statistics form a classical limit for quantum theories (Lukash 1980; Chibisov \& Mukhanov 1982; Grishchuk 1995) and is compatible with the gravitational waves theory (Abbott \& Harari 1986; Allen 1996; Allen \& Romano 1999; Maggiore 2000). Probability and appropriate expectation values may depend on the wave frequency, but not on the direction of propagation, neither the wave phase at any time or position. A random choice of plane waves guarantees that the perturbations and their canonical momenta are statistically independent and uncorrelated quantities at any time. This finally results in the stability of homogeneous expanding environment. Perturbations larger than the sound horizon decay during the radiation era, while those well inside the horizon keep their magnitude constant in time. This property, although contradicting Jeans conjecture, confirms results obtained in other gauges: synchronous (Grishchuk 1994; Golda \& Woszczyna 2001), longitudinal (Mukhanov et al. 1992 Part III) and in Hamiltonian formalism (Chibisov \& Mukhanov 1982). Similar decrease in amplitude occurs for the large scale component of the scalar field (Stebbins \& Veerarghavan 1993).

Cosmic structure formation naturally occurs at the transition from the radiational to matter domination era. To match the acoustic field at the decoupling, the growing and decaying modes contribute in the short wave limit with nearly opposite phases, and therefore, compensate each other at $\Sigma$. After the transition both modes "decouple" and the resulting superposition grows explosively. Physically it means that the pressure decay from $p=\epsilon / 3$ to $p=0$ excite perturbations much lower than horizon scale, while leave untouched the amplitude of those, which substantially exceed horizon ${ }^{10}$. For growth of inhomogeneities the break down of the acoustic approximation is responsible, i.e. the same group of physical phenomena that may excite shock waves in the interstellar medium. This kind of instabilities cannot be described by any formalism, which a priori neglects the role of decaying modes, no matter how

\footnotetext{
${ }^{10}$ This behaviour is opposite to that of tensor perturbations. A similar transition in the equation of state will amplify large scale gravitational waves not affecting the small scale ones.
}

realistic are the models for physics of recombination and decoupling, which are used.

This is obvious that the sharp transition between cosmological eras is not a realistic model for decoupling or recombination. In reality these phenomena are continuous, take some cosmologically substantial time, and involve a number of complex physical processes. The recombination and decoupling do not coincide. One can hardly expect that the realistic situation can be described by simple analytical solutions as presented in this paper. Eventually one have to apply numerical codes involving multi fluid hydrodynamics or magnetohydodynamics. Still, the problem of the initial state remains. The physical meaning of the obtained numerical results strongly depends on their stability against initial condition and on the physical relevance of the initial state assumed at early epochs. Most of hydrodynamical codes are ready to work with travelling waves, therefore, the complete numerical analysis of random acoustic fields in the expanding universe - without neglecting a priori the role of "decaying modes" - is basically possible. On the other hand, simple but nontrivial analytic solutions presented in this paper may easily be used to verify numeric procedures.

Acknowledgements. We thank Zdzisław Golda for valuable critical comments. This work was partially supported by State Committee for Scientific Research, project No. 2 P03D 01417.

\section{References}

Abbott, F. L., \& Harari, D. D. 1986, Nucl. Phys. B, 264, 487

Allen, B., Flanagan, E., \& Papa, M. A. 2000, Phys. Rev. D, 61, 24024

Allen, B. 1996 [gr-qc/9604033]

Allen, B., \& Ottewill, A. C. 1997, Phys. Rev. D, 56, 545

Allen, B., \& Romano, D. 1999, Phys. Rev. D, 59, 102001

Anderson, T. W. 1971, The statistical analysis of time series (New York: John Wiley \& Sons)

Bardeen, J. M. 1980, Phys. Rev. D, 22, 1882

Birrell, N. D., \& Davies, P. C. W. 1982, Quantum Fields in Curved Space (Cambridge: Cambridge University Press)

Brandenberger, R., Kahn, R., \& Press, W. H. 1983, Phys. Rev. D, 28, 1809

Bose, S., \& Grishchuk, L. P. 2002, Phys. Rev. D, 66, 43529

Chibisov, G. V., \& Mukhanov, V. F. 1982, MNRAS, 200, 535

Darmois, G. 1927, Mémorial de Sciences Mathématiques, Fasc XXV, Les Équations de la Gravitation Einsteinienne (Paris: Gauthier-Villars)

Deruelle, N., \& Mukhanov, V. F. 1995, Phys. Rev. D, 52, 5549

Ellis, G. F. R., \& Bruni, M. 1989, Phys. Rev. D, 40, 1804

Ellis, G. F. R., Bruni, M., \& Hwang, J. 1990, Phys. Rev. D, 42, 1035

Ellis, G. F. R., Hellaby, C., \& Matravers, D. R. 1990, ApJ, 364, 400

Fang, L. Z., \& Wu, X. P. 1996 [arcXiv:astro-ph/9601087]

Field, G. B., \& Shepley, L. C. 1968, Ap\&SS, 1, 309

Frieman, J. A., \& Turner, M. 1984, Phys. Rev. D, 30, 265

Golda, Z., \& Woszczyna, A. 2001, Class. Quantum Grav., 18, 543 [gr-qc/0002051]

Golda, Z., \& Woszczyna, A. 2001, J. Math. Phys., 42, 856

Grishchuk, L. P. 1974, JETP, 67, 825

Grishchuk, L. P. 1994, Phys. Rev. D, 50, 7151

Grishchuk, L. P. 1995 [gr-qc/9511074]

Grishchuk, L. P. 1996 [gr-qc/9603011]

Grishchuk, L. P. 1998 [gr-qc/9801011]

Hannestad, S. 2000 [arXiv: astro-ph/0311491] 
Hawking, S. W., \& Ellis, G. F. R. 1973, The Large Scale Structure of Space-Time (London: Cambridge University Press)

Hu, W. 1998, Phys. Rev. D, 59, 21301

Hwang, J., \& Vishniac, E. T. 1991, ApJ, 382, 363

Israel, W. 1966, Nuovo Cimento, 44B, 1 and corrections in ibid, 48B, 463

Jackson, J. C. 1993, MNRAS, 264, 729

Kodama, H., \& Sasaki, M. 1984, Prog. Theoret. Phys. Suppl., 78, 1

Khoperskov, A. V., \& Khrapov, S. S. 1999, A\&A, 345, 307

Liang, E. T. P. 1977, MNRAS, 180, 117

Lichnerowicz, A. 1955, Theories relativistes da la gravitation et de l'electromagnetisme (Paris: Masson)

Loeve, M. 1963, Probability theory (Princeton: Princeton University Press)

Lukash, V. N. 1980, Sov. Phys. JETP, 79, 1601

Lukash, V. N. 1999 [arXiv:astro-ph/9910009]

Lyth, D. H., \& Mukherjee, M. 1988, Phys. Rev. D, 38, 485

Lyth, D. H., \& Stewart, E. D. 1990, ApJ, 361, 343

Maggiore, M. 2000, ICTP Lecture, June 2000 [gr-qc/0008027]

Montenegro, L. E., Yuan, C., \& Elmegreen, B. G. 1999, ApJ, 520, 592

Mukhanov, V., Feldman, H., \& Brandenberger, R. 1992, Phys. Rep., 215,203

Oliveira-Costa, A., Tegmark, M., Zaldarriaga, M., et al. 2003 [arXiv:astro-ph/0307282]
Olson, D. W. 1976, Phys. Rev. D, 14, 327

Padmanabhan, T. 1993, Structure Formation in the Universe (Cambridge: Cambridge University Press)

Parker, L. 1972, Phys. Rev. D, 5, 2905,

Peebles, P. J. E. 1980, The Large Scale Structure of the Universe, Princeton Series in Physics (New Jersey: Princeton)

Press, W. H., \& Vishniac, E. T. 1980, ApJ, 236, 323

Riazuelo, A., \& Deruelle, N. 2000, Ann. Phys., 9, 288

Sachs, R. K., \& Wolfe, A. M. 1967, ApJ, 147,73

Sakai, K. 1969, Prog. Theoret. Phys., 41, 1461

Sobczyk, K. 1991, Stochastic differential equations (Kluwer Academic Publishers B. V.)

Stebbins, A., \& Veerarghavan, S. 1993, Phys. Rev. D, 48, 2421

Stone, M. 2000, Phys. Rev. D, 62, 1341

Stoeger, W. R., Ellis, G. F. R., \& Schmidt, B. G. 1991, Gen. Rel. Grav., 23, 1169

White, P. C. 1973, J. Math. Phys., 14, 831

White, M. 1992, Phys. Rev. D, 46, 4198

Whitham, G. B. 1974, Linear and Nonlinear Waves (New York: Wiley)

Woszczyna, A., \& Kulak, A. 1989, Class. Quantum Grav., 6, 1665

Wu, K., Lahav, O., \& Rees, M. 1989, Nature, 397, 225

Voglis, N. 1986, A\&A, 165, 10

Yaglom, A. M. 1961, Second order homogeneous random fields, in Fourth Berkeley Symp., II (University of California Press) 